\title{
Diminution pour le titre de formation postgraduée «Médecin praticien»
}

\section{Patrick Müller}

Chef de la division Médecine et tarifs ambulatoires

Le $1^{\text {er }}$ janvier 2018, le Conseil fédéral est intervenu une deuxième fois dans le TARMED avec des mesures très détaillées. Une des mesures ordonnées par le Conseil fédéral concerne l'introduction d'un facteur de valeur intrinsèque uniforme et celle d'un facteur d'échelle pour les porteurs du titre de formation postgraduée de médecin praticien. L'application de ce facteur de réduction a soulevé de nombreuses questions.

Suite à l'échec de la révision du tarif par les partenaires tarifaires à l'été 2016, le Conseil fédéral a fait une deuxième fois usage de sa compétence subsidiaire et est intervenu de façon approfondie dans le TARMED au $1^{\mathrm{er}}$ janvier 2018. Les détails des mesures ont déjà été présentés dans le Bulletin des médecins suisses [1]. Une de ces mesures concerne l'introduction d'un facteur de valeur intrinsèque uniforme de 0,985 et donc le nivellement des valeurs intrinsèques quantitatives. Désormais, le barème de coûts par minute de la prestation médicale (PM) ne dépend plus de la formation postgraduée suivie ni de la position tarifaire. A une exception notable: pour le titre de formation postgraduée de médecin praticien, le Conseil fédéral a introduit un facteur d'échelle obligatoire (réduction) de 0,93 sur la PM (basé sur les nouveaux points tarifaires PM de la version 1.09_BR du TARMED). Cette réduction s'applique à tous les médecins qui sont exclusivement en possession du titre fédéral de formation postgraduée de médecin praticien et qui n'ont obtenu aucun titre de spécialiste. Comme l'OFSP, chargé de la mise en œuvre technique de l'ordonnance, n'a donné aucune définition précise concernant l'application de ce facteur, la FMH a été très sollicitée ces dernières semaines par des médecins souhaitant notamment savoir ce qui est exactement entendu par «médecin praticien» et comment ce facteur est appliqué.

\section{Titre de formation postgraduée «Médecin praticien"}

Créé par la Suisse suite à la signature des accords bilatéraux avec l'UE et mis en vigueur en 2002, le titre fédé- ral de formation postgraduée de médecin praticien permet, au terme de six ans d'études de médecine et de seulement trois ans de formation postgraduée sanctionnés par un examen, d'obtenir une autorisation cantonale d'exercer et donc de pouvoir exercer une activité médicale indépendante dans toute la Suisse. Les médecins qui ont obtenu, dans un Etat membre de l'UE, un titre de spécialiste en médecine générale inscrit dans la directive UE 2005/36 au point 5.1.4 «Titres de formation de médecin généraliste» ne peuvent prétendre en Suisse qu'au titre de médecin praticien en vertu de l'accord sur la libre circulation des personnes Suisse/UE. Lors de la procédure de reconnaissance des titres de généraliste étrangers, la Commission des professions médicales (MEBEKO) de l'Office fédéral de la santé publique (OFSP) ne tient pas compte de la durée de la formation postgraduée suivie (5 ou 3 ans). Dans tous les cas, le titre de médecin praticien n'équivaut expressément pas à un titre de spécialiste et ne permet par exemple pas d'obtenir une attestation de formation complémentaire.

Le titre de formation postgraduée de médecin praticien ne doit pas être confondu avec la dénomination «méd. pract.». Par le passé, cette dénomination était souvent utilisée par les médecins qui n'avaient pas obtenu le titre de docteur. Cette dénomination n'a aucun lien avec la formation postgraduée suivie ni le titre de spécialiste obtenu; elle indique uniquement que le médecin n'a pas présenté de thèse. En raison du risque de confusion avec le titre de médecin praticien, la FMH recommande aujourd'hui de ne plus utiliser cette dénomination et d'utiliser à la place celle de «médecin diplômé». 


\section{Quand appliquer le facteur d'échelle?}

Selon l'ordonnance du Conseil fédéral, les médecins qui possèdent uniquement le titre de formation postgraduée de médecin praticien doivent appliquer le facteur d'échelle de 0,93 sur la prestation médicale (PM, points tarifaires) de toutes les positions tarifaires fournies. Le nombre de points tarifaires de la PM est donc diminué pour chacune d'entre elles, y compris pour les positions tarifaires relevant des droits acquis du médecin. Le facteur doit figurer de manière transparente sur la facture au niveau de la position tarifaire (champ fPM du formulaire de facturation XML). Les médecins qui, en plus du titre de médecin praticien, possèdent aussi un ou plusieurs titres de spécialiste fédéraux ou formellement reconnus par la Suisse («porteurs de plusieurs titres») ne sont pas concernés par cette réduction et ne doivent donc appliquer ce facteur pour aucune position (prestations de la médecine de premier recours incluses).

Correspondance FMH / Division Médecine et tarifs ambulatoires

Baslerstrasse 47 CH-4600 Olten

Tél. 0313591230

Fax 0313591238

tarife.ambulant[at]fmh.ch
Lorsqu'une facture comprend à la fois des prestations fournies par un médecin praticien et des prestations fournies par un spécialiste, le facteur d'échelle ne s'applique que sur les prestations fournies par le médecin praticien. Cela vaut également pour les factures qui sont établies avec un numéro RCC commun (p. ex. par
Que faire lorsqu'une facture est rejetée en raison de l'absence du facteur

\section{d'échelle?}

Nous avons appris que les assureurs vérifiaient scrupuleusement I'application correcte du facteur d'échelle. Si une facture est retournée par une assurance au motif que le facteur d'échelle n'a pas été appliqué, les possibilités qui s'offrent à vous sont les suivantes:

- Vérifier les données du médecin concerné dans le registre des professions médicales: www.medregom.admin.ch.

- Si un titre de spécialiste est inscrit dans le registre, la facture a été refusée à tort. Dans ce cas, il faut vous adresser à la société SASIS SA, qui est chargée de tenir la liste des titres de formation postgraduée pour les assureurs, ou clarifier la question directement avec l'assureur.

- Pour toute autre question (p. ex. lorsqu'aucun titre de formation postgraduée ne figure dans le registre), vous pouvez vous adresser à la division Médecine et tarifs ambulatoires à l'adresse tarife.ambulant[at]fmh.ch.

une institution ou un cabinet de groupe); dans tous les cas, c'est le médecin qui fournit la prestation (position tarifaire) qui est déterminant.

Références

1 Bull Méd Suisses, 2017;98(47):1568-71 Bull Méd Suisses, 2017;98(28-29):888-91 